Kansas State University Libraries

New Prairie Press

\title{
MARKOV-RECAPTURE POPULATION ESTIMATES
}

E. Paul Wileyto

Follow this and additional works at: https://newprairiepress.org/agstatconference

Part of the Agriculture Commons, and the Applied Statistics Commons

\section{(c) (†) $\ominus$}

This work is licensed under a Creative Commons Attribution-Noncommercial-No Derivative Works 4.0 License.

\section{Recommended Citation}

Wileyto, E. Paul (1994). "MARKOV-RECAPTURE POPULATION ESTIMATES," Conference on Applied

Statistics in Agriculture. https://doi.org/10.4148/2475-7772.1355

This is brought to you for free and open access by the Conferences at New Prairie Press. It has been accepted for inclusion in Conference on Applied Statistics in Agriculture by an authorized administrator of New Prairie Press. For more information, please contact cads@k-state.edu. 


\title{
MARKOV-RECAPTURE POPULATION ESTIMATES
}

\author{
E. Paul Wileyto \\ U.S.D.A., A.R.S. \\ U.S. Grain Marketing Research Laboratory \\ 1515 College Avenue \\ Manhattan, KS 66502
}

\begin{abstract}
This paper reviews recent development of a method for estimating insect populations. It is like mark-recapture methods, except that marking is done passively at bait stations by the insects themselves, and capture probabilities are generated using a simple Markov process model. Assumptions about rates of marking and capture are made from the sampling scheme, and the estimate is based upon the resulting multinomial probability distribution and maximum likelihood methods. The paper continues to review the sampling distributions for the population estimate, revealed by simulation, and explores correction of the bias. Relative likelihood based confidence intervals are compared with two standard error intervals, and found to perform better over a wide range of parameter values, especially where the number of recaptures is small. The method tends to become biased when used in an open or growing population. Goodness of fit tests are possible with the added degrees of freedom, but are not very powerful.
\end{abstract}

\section{Key Words}

Trap; population; estimate; markov process; mark-recapture; likelihood, relative likelihood interval, profile likelihood interval.

\section{Introduction}

Trapping is the usual method for monitoring insect populations because it is simple to use. It has generally provided only presence/absence information because of the unspecifiable relationship between population size and trap catch. Traditional methods of population estimation, including mark-recapture and removal, are generally too labor intensive to use with insect populations. For mark-recapture, the initial episode involves either mass rearing of insects for release, or capture with arduous care to maintain subjects in good condition. This intense labor is often followed by few or no recaptures. Likewise, removal methods are too labor-intensive for insect populations, because they require several sequential visits over to the traps over a short period in order to obtain an estimate.

We have developed an alternative which involves only the placement of traps, 
yet provides a mark-recapture type of estimate from a single trap observation by allowing subjects to mark themselves (Wileyto et al. 1994, Wileyto 1994). Although the primary application is the estimation of closed insect populations, the method could be useful for many other species. In order to use the technique, some of the traps must be converted into passive marking stations where subjects may visit and leave marked, while the remaining half continues to capture insects in the usual way. We have made such bait stations using commercially available wing or pitfall traps baited with food or pheromone lures; they differ in that they are filled with a fluorescent dye and modified to allow escape.

The statistical interpretation of self-marking was accomplished by first making explicit assumptions about how the system works. One assumption is that the population is closed (apart from trapping). Another is that we know the relative (percapita) rates of marking and capture. Secondly, we generated capture probabilities by modelling self-marking and capture as a markov process. The following describes two probability models relating to the events of capture and recapture under the assumptions described above. This is followed by Monte-Carlo tests of several important characteristics of the population estimate, and a discussion of the confidence limits.

\section{Sampling Schemes and Models}

A closed population of $\mathrm{N}$ individuals, exposed to marking stations and traps, is involved in a Markov process (Wileyto et al. 1994, Wileyto 1994). All $(N)$ individuals begin in the free and unmarked state (frequency $F$ ), and as the trapping proceeds, population members may remain there or be distributed to any of the other states: free and marked (frequencies denoted as $M$ with various subscripts), unmarked and captured (frequency denoted $C$ ), or (variously) marked and captured (denoted $R, Q, S$ ) (Fig. 1). The per capita trapping rate is defined as $\lambda$. Per capita marking rates for a particular marking category depend upon $\alpha, \beta, \ldots$ the ratios of marking stations to traps, and the rate is the multiple $\alpha \lambda$. For example, if there are two red marking stations per trap, the per capita marking rate for red is $2 \lambda$. These models are most easily analysed as a series of differential equations, describing the input and loss rates for each category. The overall rates of gain and loss are simply per capita rates times the principal. For example, if category $F$ has a per capita loss rate of $3 \lambda$, the total loss rate is $3 \lambda F$.

I will begin with the most complicated model we use (double marking), because it is general, and simpler models follow directly from it. Suppose we have two marking station types per trap, at a rate of $\alpha$ marking stations per trap of one type (eg. color = red), and $\beta$ of another type (eg. color = blue). The result is a series of eight differential equations which describe the expected trajectories for all eight frequencies (Figure 1): 


$$
\begin{array}{ll}
E(F)^{\prime}=-(1+\alpha+\beta) \lambda F & E(C)^{\prime}=\lambda F \\
E\left(M_{1}\right)^{\prime}=\alpha \lambda F-(1+\beta) \lambda M_{1} & E(R)^{\prime}=\lambda M_{1} \\
E\left(M_{2}\right)^{\prime}=\beta \lambda F-(1+\alpha) \lambda M_{2} & E(Q)^{\prime}=\lambda M_{2} \\
E\left(M_{3}\right)^{\prime}=\beta \lambda M_{1}+\alpha \lambda M_{2}-\lambda M_{3} & E(S)^{\prime}=\lambda M_{3} .
\end{array}
$$

Substituting $e^{-\lambda t}=p$ yields multinomial probabilities for $\mathrm{N}$ trials:

$$
\begin{aligned}
& \operatorname{PI}(\text { UNOBSERVED })=p \\
& \operatorname{Pr}\left(\begin{array}{c}
\text { UNMARKED } \\
\text { TRAPPED }
\end{array}\right)=\frac{1-p^{1+\alpha+\beta}}{1+\alpha+\beta} \\
& \operatorname{Pr}\left(\begin{array}{c}
M_{1} \\
\operatorname{eg}, \operatorname{TrAPPED}
\end{array}\right)=\frac{\alpha}{(1+\beta)(1+\alpha+\beta)}-\frac{p^{1+\beta}}{1+\beta}+\frac{\left.p^{1+\alpha+\beta}\right)}{1+\alpha+\beta} \\
& \operatorname{Pr}\left(\begin{array}{c}
M_{2} \operatorname{TRAPPED} \\
\operatorname{eg} \cdot b l u e
\end{array}\right)=\frac{\beta}{(1+\alpha)(1+\alpha+\beta)}-\frac{p^{1+\alpha}}{1+\alpha}+\frac{\left.p^{1+\alpha+\beta}\right)}{1+\alpha+\beta} \\
& \operatorname{Pr}(\underset{\text { TRARKED }}{\text { MOUBLY }})=\frac{\alpha \beta(2+\alpha+\beta)}{(1+\alpha)(1+\beta)(1+\alpha+\beta)} \\
& -p+\frac{p^{1+\alpha}}{1+\alpha}+\frac{p^{1+\beta}}{1+\beta}-\frac{p^{1+\alpha+\beta}}{1+\alpha+\beta} \text {. }
\end{aligned}
$$

(Note that $F$ (free, unmarked) and $M$ (free, marked) frequencies have been lumped to form a single unobserved frequency.) The resulting model has four degrees of freedom.

The model is simpler if there is only one type of mark, for instance red. In this case, $\beta$ is 0 , and the capture probabilities reduce to:

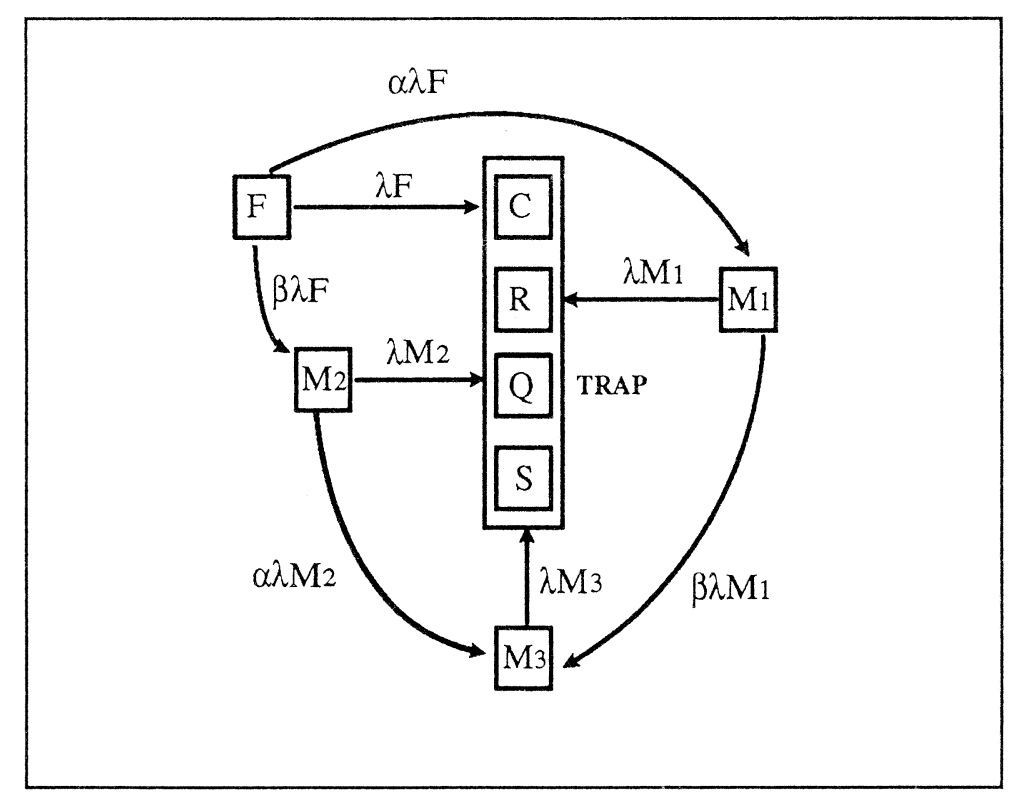

Fig. 1. The general double marking scheme. There are $\alpha$ red marking stations and $\beta$ blue marking stations per trap. 


$$
\begin{aligned}
\operatorname{Pr}(\text { UNOBSERVED }) & =p \\
\operatorname{Pr}\left(\begin{array}{c}
\text { UNMARKED } \\
\text { CAPTURE }
\end{array}\right) & =\frac{1-p^{1+\alpha}}{(1+\alpha)} \\
\operatorname{Pr}\left(\begin{array}{c}
\text { MARKED } \\
\text { CAPTURE }
\end{array}\right) & =\frac{\alpha-p-\alpha p+p^{1+\alpha}}{(1+\alpha)}
\end{aligned}
$$

Where traps and marking stations are used at a 1:1 ratio, the capture probabilities reduce to those of the original work (Wileyto et al. 1994):

$$
\begin{aligned}
\operatorname{Pr}(\text { UNOBSERVED }) & =p \\
\operatorname{Pr}\left(\begin{array}{c}
\text { UNMARKED } \\
\text { CAPTURE }
\end{array}\right) & =\frac{1-p^{2}}{2} \\
\operatorname{Pr}\left(\begin{array}{c}
\text { MARKED } \\
\text { CAPTURE }
\end{array}\right) & =\frac{(1-p)^{2}}{2} .
\end{aligned}
$$

\section{Estimation Procedures}

Point estimation is accomplished by a straightforward application of maximum likelihood techniques. The parameters $\alpha$ and $\beta$ are assumed, referring to the relative numbers of marking stations in the specific sampling scheme. The parameters $N$ and $p$ are estimated simultaneously. The solution for $\hat{N}$ from multinomial capture data is determined from the likelihood $(L)$ by setting $L(N, p)=L(N-1, p)$, and is well known as:

$$
\hat{N}=\frac{\text { Total Caught }\{C+R+\ldots\}}{1-\hat{p}}
$$

(See Seber 1982, Burnham et al. 1987). The solution of $\hat{p}$ comes from setting

$$
\frac{\partial \ln L}{\partial p}=0
$$

where $\ln L$ is the $\log$ of the likelihood function, either analytically or numerically. (See Pollard (1977) for a review of methods.)

Allowable values for $\hat{p}$ fall between zero and one, although certain data combinations will result in a negative solution for $\hat{p}$, and population estimate (3) which is smaller than our sample. (When the maximum likelihood estimate of $p$ is negative, there will be a local (constrained) maximum of the likelihood function at the 
boundary $p=0$. Under such circumstances, the boundary estimate (constrained maximum) where $\hat{p}=0$, and $\hat{N}=\{$ Total Captured $\}$, should be accepted.

The solution for $\hat{p}$ cannot easily be determined explicitly under most sampling schemes, and should be found numerically. However, solutions for $\hat{p}$ arising from (4) under specific schemes may often be characterized explicitly, using Descartes' rule and related methods (see Pearson 1990). For example, for all specific schemes used in this paper, it is possible to determine boundaries between data combinations that yield a single solution for $\hat{p}$ between zero and one, and those which yield only negative solutions. This allows the user to anticipate boundary solutions, or other unusual results.

\section{Bias of the Estimate}

Though maximum likelihood estimates are asymptotically unbiased, they are often biased for smaller samples. Bias is defined as the difference between the mean value of the estimate of a parameter and the true value of the parameter:

$$
B I A S=E(\hat{N})-N
$$

The mean of $\hat{N}$ cannot be obtained from the multinomial probability distribution unless $\hat{N}$ has a simple analytical form; the mean can be approximated by simulation.

Estimation and reduction of bias for an individual sample may be accomplished through the jackknife technique (Potvin and Roff 1993, Hinkley 1983, Efron 1982). The technique uses a systematic resampling of $K$ observations by sequentially dropping one observation at a time, to produce $K$ samples of $K-1$. The estimate of bias is then

$$
\begin{aligned}
B I A S & =-\frac{K-1}{K} \sum_{K}\left(\hat{N}_{K}-\hat{N}_{K-1}\right) \\
& =-\frac{K-1}{K}\left[C\left(\hat{N}_{C}-\hat{N}_{C-1}\right)+r\left(\hat{N}_{I}-\hat{N}_{r-1}\right)+. . . . .\right]
\end{aligned}
$$

Efron (1982) suggests a corresponding correction in which each of the $K$ observations is added (duplicated) in turn, to produce a slightly different estimate of bias:

$$
\begin{aligned}
\text { BIAS } & =\frac{K+1}{K} \sum_{K}\left(\hat{N}_{K}-\hat{N}_{K+1}\right) \\
& =\frac{K+1}{K}\left[C\left(\hat{N}_{C}-\hat{N}_{C+1}\right)+r\left(\hat{N}_{r}-\hat{N}_{r+1}\right)+\ldots . . .\right] .
\end{aligned}
$$

This estimate of bias has an advantage when the probability of capture in several categories is small. We cannot generate estimates using the Markov-recapture 
technique if too many categories have zero entries, and having an entry of one in several categories would then disable the standard jackknife method. The method described by (5) is not disabled by such data.

\section{Confidence intervals}

Two approaches were tested for determining confidence limits around the estimate of $N$. The first approach is through the theoretical approximation of variances of the estimators (3) and (4), which are obtained from the inverse of the information matrix (Mood et al. 1974, Lehmann 1983), found by taking the second derivatives of the log-likelihood functions. For algebraic approximations, $N$ and $p$ are substituted by estimates $\hat{N}$ and $\hat{p}$ respectively. An approximate 95 percent confidence interval for $\mathrm{N}$ may then be obtained as

$$
\text { C.I. }(95)_{\hat{N}}=\hat{N} \pm 2 \sqrt{\operatorname{VAR}(\hat{N})} \text {. }
$$

The two standard error approach at times produces a confidence interval which misses the true parameter value more often than it should. A more recent approach is the relative likelihood interval (also called a "profile likelihood" interval- See Arnason et al. 1991, Lebreton et al. 1992). The approach bases the upper and lower limits on the likelihood ratio $\left(L R=L(\hat{N}, \hat{p}) / L\left(N, p^{*}\right)\right)$, where $p^{*}$ is the maximum likelihood estimate of $p$, given $N)$. Since $2 \operatorname{Ln}(L R)$ is distributed approximately as $\chi^{2}$ with one degree of freedom, limits occur where

$$
-2 \operatorname{Ln}(L R)=3.84,
$$

the 95 percent significance value for a two sided chi-square test. Relative likelihood intervals tend to be as broad as the corresponding two standard error interval, although the location differs, and the interval may be asymmetric about the estimate (Lebreton et al. 1992).

\section{Specific Models for Testing}

In the preceding, I have outlined the general procedures for estimating population size and confidence limits based upon generalizations of the Markovrecapture sampling scheme. I now address the performance of these procedures. First, I must specify models by providing values for marking rates $\alpha$ and $\beta$ in (1) and (2). (Generally, the algebra simplifies greatly when integer values are substituted for $\alpha$ and $\beta$.) I generated three specific sets of capture probabilities for the following trapping schemes: A) single mark, $\alpha=1$, with one marking station per trap, B) single mark, $\alpha=2$, with two similar marking stations per trap, C) double mark, $\alpha=1, \beta=1$, with two dissimilar marking stations per trap, and D) double mark, $\alpha=2, \beta=1$, with three 
marking stations per trap, two of one color and one of another. Model A has one added virtue in an analytical form for the population estimate:

$$
\hat{N}=\frac{(C+R)^{2}}{2 R}
$$

and a virtually unbiased variant:

$$
\hat{N}=\frac{(C+R)^{2}}{2(R+1)}
$$

Following Descartes' rule in examining the partial derivatives with respect to $\mathrm{p}$, Model A has one real positive solution as long as $R<C$, and that solution falls between zero and one. If $R>C$, all real solutions for $\hat{p}$ are negative, and the solution for $\hat{N}$ will be less than the observed number. ( $R \leq C$ represents a boundary for reasonable estimates of $\hat{p}$ and $\hat{N}$.) When $R$ falls above $C$, there is a local maximum in the sample space (boundary estimate), where

$$
\hat{\rho}=0, \quad \hat{N}=C+R .
$$

This estimate should be used whenever $R>C$. Model $\mathrm{B}$, likewise, has one real positive solution between zero and oneas long as $R<2 C$. Model $C$ violates the boundary if $2 S>C+R+Q$. All solutions for $\mathrm{p}$ are then negative, and the boundary estimate should be used:

$$
\hat{p}=0, \quad \hat{N}=C+R+\ell+S .
$$

Likewise, model D violates the boundary if $S \geq(5 / 7)(C+R+Q)$.

\section{Properties of Population Size Estimates}

In order to examine the properties of the above estimates and confidence limits, I simulated trap results. In each simulation, the $N$ population members were probabilistically allocated to each of the categories (unobserved, unmarked, and various marking categories) according to their respective probabilities (1) and (2). The value of $N$ was either 250 or 1000 . Values of $p$ were selected between .975 and 2 . Generally, I did 5000 trials for each parameter combination. Trials with zero recaptures (summed for all recapture classes) were repeated. I generated an estimate (3) and a jackknifed estimate ((3)-(5)) for each set of trap results. Where numerical methods were required, I used the Newton-Raphson technique based on numerical 
derivatives (Pollard 1977). I recorded the average of both estimates for each parameter combination.

The performance of confidence intervals was evaluated using the simulated trap data described above. The standard measures of performance are the 1) average length of the confidence interval, and 2) the frequency with which the interval included the true value of $N$ (coverage) (Arnason et al. 1991). I recorded each measure for both the two standard error interval (evaluated using (6), and the jackknife corrected estimates) and the relative likelihood interval (7). I used the technique developed by Venzon and Moolgavcar (1988) to find the two solutions to (7), with starting points at the upper two standard error limit (6) on the upper side, and just below the estimate of $N$ (3) on the lower side.

I now present the results of those simulations, beginning with an examination of bias. Means from simulations of single marking (Models A and B) for populations of 250 and 1000 are shown in Figures $2 \mathrm{a}$ and b. They are summarized as follows. For extremely large values of $p$ (little progress towards trapping), the mean estimate falls below the true value. For slightly lower values of $p$, the mean rises sharply to where it is approximately 30 percent higher than the true value. For lower values of $p$, the mean falls and asymptotically approaches the true value of $N$. Overall, the 2:1 scheme is less biased than the 1:1 scheme, over a wide range of $p$ values. The peak bias is approximately the same, about 30 percent high, but it occurs after much less trapping time. This implies that the $2: 1$ marking scheme will provide useable estimates from smaller samples (less trapping effort) than will the 1:1 scheme.

Figure $2 c$ shows results of double marking at a rate of 1:1:1 (Model C). The pattern observed while varying the value of $p$ is similar to that of the single marking scheme, but estimates are generally less biased than their single marking counterparts, with a peak bias nearly 20 percent higher than the true value (compared to 30 percent high for single marking). Finally, the results of double marking at a rate of 2:1:1

(Model D) are shown in Figure $2 \mathrm{~d}$. The mean of the unadjusted estimate shows the usual positive bias at higher values of $p$. The peak bias seems to be a bit smaller than either of the other schemes tested, at 12-15 percent high, and the bias remains below that of Model $\mathrm{C}$, all due to the increase in the total marking rate.

Jackknife corrected estimates (Fig. 2) have a negative bias at the highest values of $p$. The reason for the negative bias at high $p$ is that the mean estimate is a conditional mean, not including observations where total recaptures equal zero, and zero recaptures is a common event at high $p$ (See Wileyto et al. 1994). We cannot do much about the negative bias at high $p$, and the estimate will remain biased until the total number of recaptures is at least five. As $p$ decreases slightly, the adjusted estimate climbs steeply to a peak only one to two percent higher than the true value, and remains almost unbiased for the remaining values of $p$. I would recommend that anyone using a Markov-recapture sampling scheme, other than the origninal 1:1 


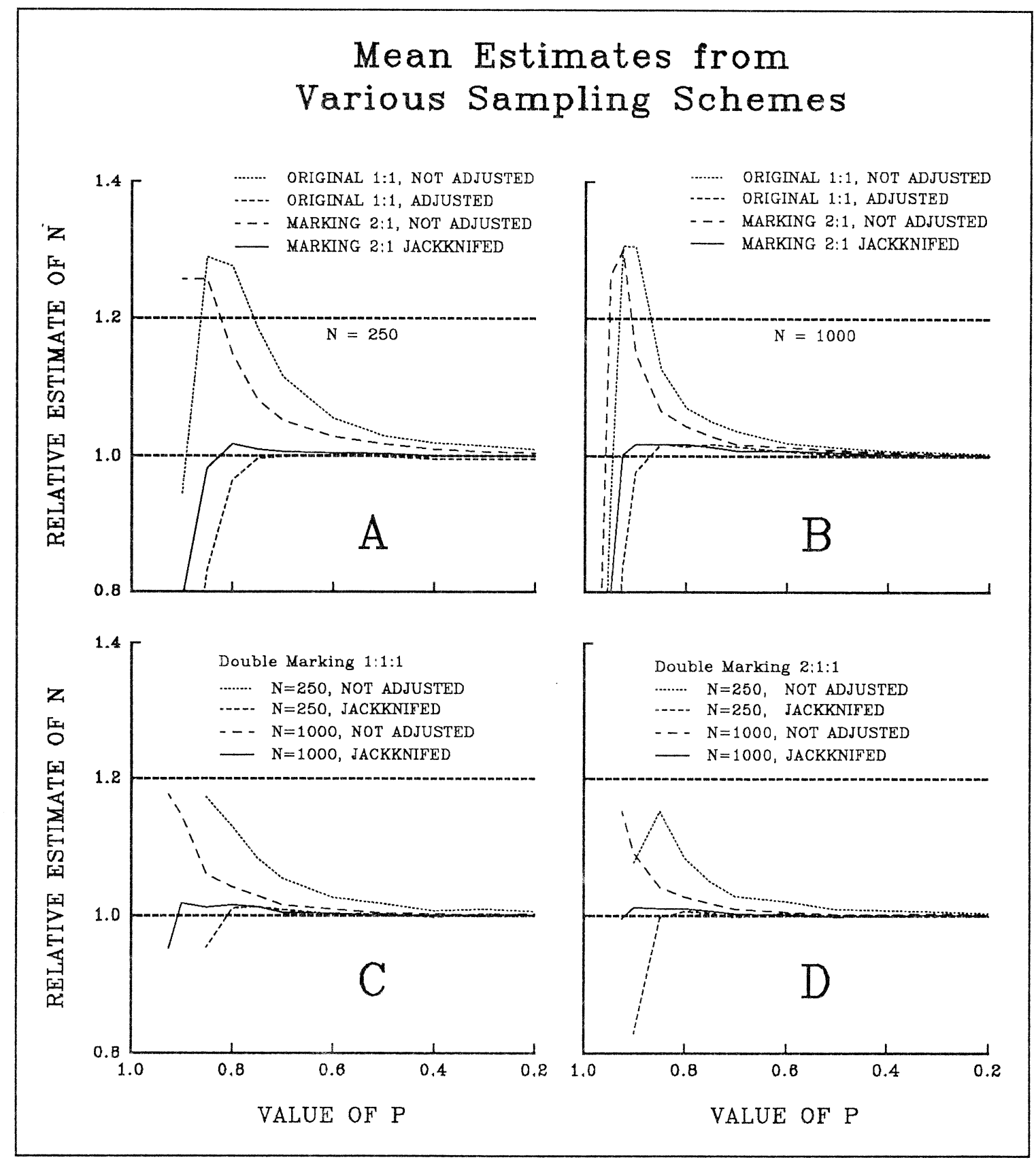

Fig. 2. Mean estimates of population size from simulations: a) single marking, original 1:1 versus $2: 1, N=250, b)$ same as a, except that $N=1000$, c) double marking, $\alpha=1, \beta=1$, and d)double marking, $\alpha=2, \beta=1$. Note that the jackknife technique of bias removal produces an almost unbiased estimate for most values of $p$.

scheme, also use the jackknife correction, while being aware of the negative bias for small numbers of recaptures. 
Moving on to discuss confidence intervals, the performance of two standard errors is revealed in Figure 3, and is summarized as follows. For very high values of $p$, which correspond to the earliest jackknife estimates that are not negatively biased, the average confidence interval length (Fig. 3a) is approximately twice the value of $N$. As $p$ decreases, the interval width first falls sharply, then continues to fall at a decreasing rate. The most clear distinctions are between the population sizes of 250 and 1000 , the larger producing a consistently narrower interval. Within each population size treatment, the single marking $(2: 1$ only shown) model gives the widest interval. (It is generally narrower than the two standard errors from original 1:1 model. The original is not shown, but it reached a similar peak at $p=0.7$ rather than $p=0.8$.) Double marking gives a narrower interval yet, with the 2:1:1 scheme giving a generally narrower interval than the 1:1:1 scheme. The pattern in coverage of two standard errors (Fig. 3b) is as follows. For high values of $p$, (similar to high values from Fig. 3a), coverage ranges from 85 to 92 percent. As $p$ decreases, coverage climbs steeply, finally approaching 95 percent

asymptotically. Populations of 1000 reached 95 percent coverage much more quickly than did populations of 250. The distinction among models was less clear for coverage than for interval width, due to noise in the simulation.

Performance tests on the relative likelihood interval reveal a pattern of interval width almost identical with that of two standard errors (Fig. 4a), except that two standard errors is slightly narrower at the highest values of $p$. (This may be a small effect of the negative bias for high p.) However, the coverage (Fig. 4b) is improved, 
staying on or around 95 percent, even for the highest values of $p$. I would therefore recommend that the relative likelihood interval be used as a 95 percent confidence interval when possible. However, I would also recognize that two standard errors provides a reasonable interval under many circumstances, and may be more practical when a computer is not available to calculate a relative likelihood interval.

\section{Field Tests}

In order to examine the performance of Markov-recapture estimates in the field, we tested the procedure on known populations of the Indianmeal moth, Plodia interpunctella. These were established in an empty warehouse by "release" of unsexed pupae. We used home-made traps constructed from disposable plastic bowls, with a pheromone lure to attract males. The bowl was filled either with a sticky insert for the trap or fluourescent dye for the marking stations. Three of each were placed in the warehouse along with the pupae. After three to five days, the traps were collected and counted (under a UV light to reveal marks).

Figure 5 reveals that the estimate compares favorably with the true value. (The true value always fell within the confidence limits.)

\section{Summary}

Markov-recapture methods provide a reduced labor method for population estimation by combining aspects of trapping and mark-recapture techniques. This is accomplished by modelling capture probabilities as a Markov-process. Several schemes and solutions have been presented and tested in this paper. The bias may be reduced for all such estimates by using the jackknife procedure, and confidence limits 
are best evaluated by the relative likelihood method. The Markov-Recapture method of population estimation can be greatly improved by placing more than one marking station per trap to increase the rate of marking. First, multiple marking stations produce an estimate inherently less biased than the original 1:1 scheme. Secondly, the increased marking reduces the confidence interval around the estimate of population size. The improvements come from obtaining more recaptures earlier in the trapping process. Additional improvements to bias and the confidence interval come from using two different kinds of marking stations, to create many more possible categories in the trap.

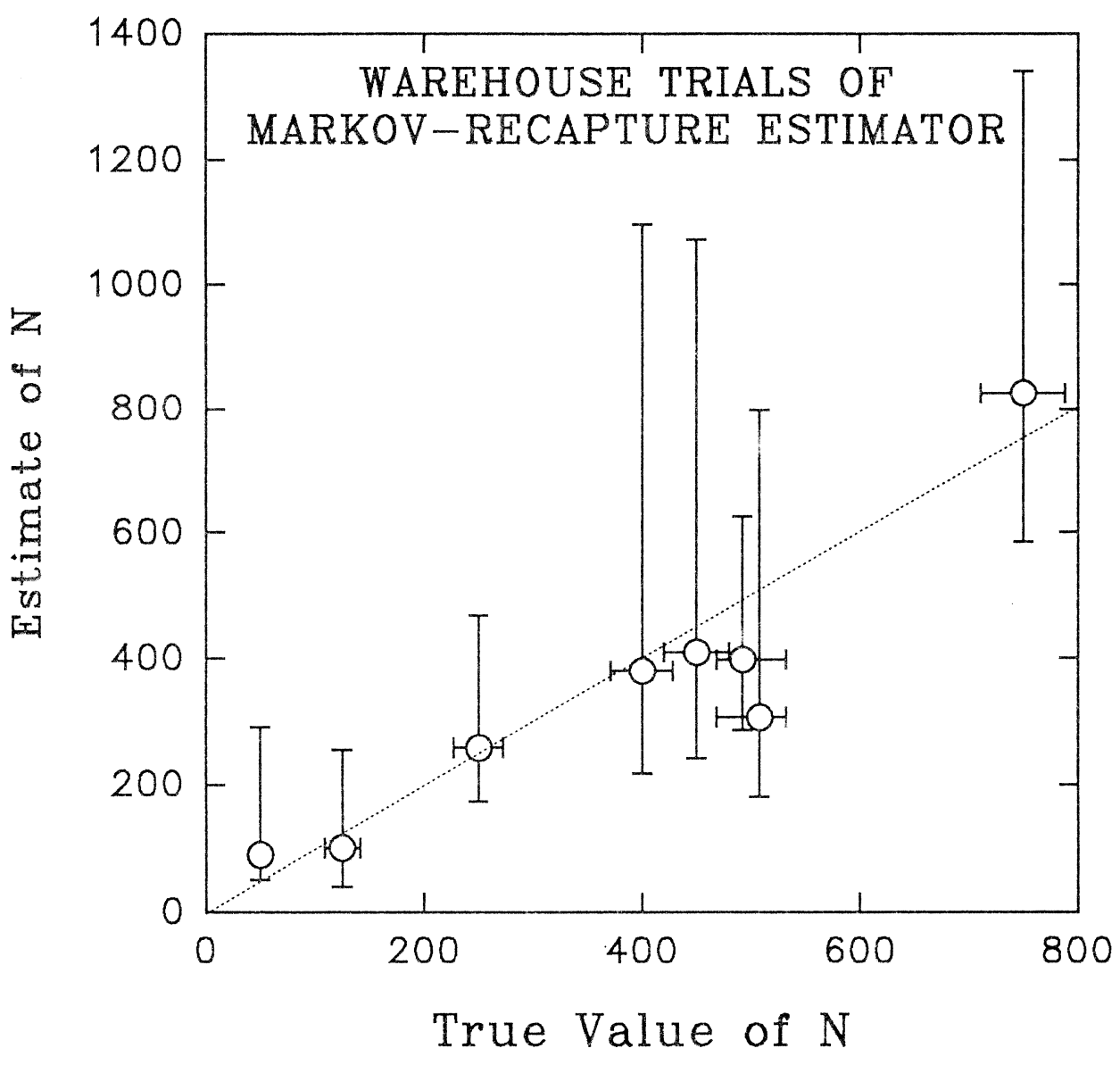

Fig. 5. Warehouse field trials of the markov-recapture estimator, using traps baited with sex attractants. Known populations of unsexed pupae were released in a warehouse. Horizontal error bars are 2 SD around the numbers of males actually present. Vertical error bars are $95 \%$ relative likelihood intervals. 
Kansas State University

\section{Acknowledgements}

Research was conducted as part of the Agricultural Research Service project on Population Ecology and Predictive Models for Packaged Commodities, CRIS No. 6605-43640-018-00D, M.A. Mullen senior investigator. I thank M.A. Mullen for his ideas which set the project in motion, and I thank W.J. Ewens for being my mentor and for introducing me to estimation problems. I am also grateful to R.T. Arbogast, J.E. Baker, W.J. Ewens, P.W. Flinn, M.A. Mullen, J.D. Nichols, L. Smith, J.E. Throne, and D.K. Weaver for their comments on this and earlier versions of this manuscript.

\section{References Cited}

Arnason, A. N., C. J. Schwarz and J. M. Gerrard. 1991. Estimating Closed Population Size and Number of Marked Animals from Sighting Data. J. Wildlife Management. 55:716-730.

Burnham, K. P., Anderson, D. R., White, G. C., Brownie, C., Pollack, K. H. 1987. Design and Analysis for Fish Survival Experiments Based on Release-Recapture. Americal Fisheries Scoiety Monograph 5. 437 pp.

Efron, B. 1982. The Jackknife, the Bootstrap and Other Resampling Plans. Regional Conference Series in Applied Mathematics. Society for Industrial and Applied Mathematics, Philadelphia, PA.

Hinkley, D. V. 1983. Jackknife Methods. Encyclopedia of Statistical Sciences. $4: 280-7$.

Lebreton, J., K. P. Burnham, J. Colbert and D. R. Anderson. 1992. Modeling Survival and Testing Biological Hypotheses using Marked Animals: A Unified Approach with Case Studies. Ecological Monographs. 62:67-118.

Lehmann, E. L. 1983. Theory of Point Estimation. Probability and Mathematical Statistics Series. Wiley, New York, NY.

Mood, A. M., F. A. Graybill and D. C. Boes. 1974. Introduction to the Theory of Statistics. Third Edition. McGraw-Hill Book Co., New York, New York.

Pearson, C. E. 1990. Handbook of Applied Mathematics: Selected Results and Methods. Second Edition. Van Nostrand Reinhold, New York, NY.

Pollard, J. H. 1977. A Handbook of Numerical and Statistical Techniques. Cambridge University Press, Cambridge, UK.

Potvin, C. and D. A. Roff. 1993. Distribution free and robust statistical methods: viable alternatives to parametric statistics? Ecology. 74:1617-1628.

Seber, G. A. F. 1982. The Estimation of Animal Abundance. Second Edition. MacMillan, New York, NY.

Venzon, D. J. and S. H. Moolgavkar. 1988. A method for computing profile-likelihood based confidence intervals. Applied Statistics. 37:87-94.

Wileyto, E. P. 1994. Improving Markov-Recapture Population Estimation with Multiple Marking. Envir. Ent. 23:1129-1137.

Wileyto, E. P., W. J. Ewens and M. A. Mullen. 1994. Markov/Recapture Population Estimates: a Tool for Improving Interpretation of Trapping Experiments. Ecology 75:1109-1117. 